\title{
Conocimiento de la morfología, conciencia morfológica y aprendizaje léxico. Objetivos “difusos” en la Enseñanza Secundaria*
}

\section{Knowledge of morphology, morphological awareness and lexical learning. "Difussed" objectives in Secondary Education}

\section{Rosa Ana Martín Vegas}

Universidad de Salamanca

rosana@usal.es

ORCID ID: https://orcid.org/0000-0002-7573-9243
DOI: $10.17398 / 1988-8430.35 .1 .107$

Fecha de recepción: $15 / 04 / 2021$ Fecha de aceptación: 13/10/2021

Martín Vegas, R. A. (2022). Conocimiento de la morfología, conciencia morfológica y aprendizaje léxico. Objetivos “difusos” en la Enseñanza Secundaria. Tejuelo, 35(1), 107-136.

Doi: https://doi.org/10.17398/1988-8430.35.1.107

\footnotetext{
* Este trabajo se ha realizado en el marco del Proyecto de Investigación titulado "Estudio del aprendizaje léxico basado en el conocimiento de la morfología del alumnado de Educación Secundaria Obligatoria. Modelo piloto de intervención didáctica" con referencia PID2020-116110GB-I00.
} 
Resumen: Este artículo es un estudio crítico del estado de la didáctica de la morfología planteado desde el desajuste entre la teoría didáctica antigramaticalista, -promulgada desde hace décadas y defendida en la actualidad sin frutos consolidados-, el currículum educativo en la Enseñanza Secundaria y las propuestas desarrolladas en los libros de texto. Se defiende la necesidad de investigación experimental que valore el impacto del conocimiento de la morfología en el desarrollo de la conciencia morfológica en adolescentes y que mida su efecto en el conocimiento y enriquecimiento del léxico. A su vez, se propone un modelo de didáctica de la morfología fundamentado en las teorías unitarias del procesamiento léxico (conexionismo y modelos asociativos) y amparado en las aportaciones de investigaciones psicolingüísticas sobre el desarrollo de la conciencia morfológica en ELE y en niños con trastornos del lenguaje. Las fases secuenciales en el desarrollo de la conciencia morfológica, así como los modelos de activación léxica regulados por los tipos de conexiones formales y semánticas y por el factor frecuencia, fundamentan los niveles didácticos del modelo. Esta propuesta debe conformarse en prácticas de aula que validen la enseñanza inductiva de la morfología orientada al desarrollo de la competencia léxica.

Palabras clave: morfología; conciencia morfológica; léxico; didáctica; enseñanza secundaria.
Abstract: This paper is a critical study of the state of the didactics of morphology raised from the mismatch between the antigramaticalist didactic theory, -promulgated for decades and currently defended without consolidated fruits-, the educational curriculum in Secondary Education and the proposals developed in textbooks. It is defended the need for experimental research to assess the impact of knowledge of morphology on the development of morphological awareness in teenagers and to measure its effect on lexical enrichment. It is proposed a morphology didactic model that is based on the unitary theories of lexical processing (connectionism and associative models) and it is supported by the contributions of psycholinguistic research on the development of morphological awareness in ELE and in children with disorders of the language. The sequential phases in the development of morphological awareness, as well as the lexical activation models regulated by the types of formal and semantic connections and by the frequency factor, define the didactic levels of the model. This proposal should be made up of classroom practices that validate the inductive teaching of morphology aimed at developing lexical competence.

Keywords: morphology; morphological awareness; lexicon; didactics; secondary education. 


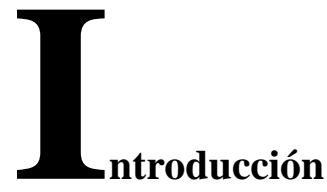

El debate en torno a la morfología como parte independiente de la gramática (modelos dualistas o conexionistas) ha generado planteamientos explicativos distintos sobre procesos de adquisición (Rumelhart y McClelland, 1986; Pinker y Prince, 1988; Plunkett y Marchman 1991, 1993; Bybee, 1991; Daugherty y Hare, 1994; Gasser, 1994, 1997) o sobre procesos diacrónicos (Dalton-Puffer, 1993; Bybee, 1995; Sánchez Miret et al., 1998; Martín Vegas, 2007), pero no ha llegado a condicionar la didáctica de la formación de palabras en la enseñanza del español como lengua materna. La atención que ha tenido la morfología en la Lingüística Aplicada ha sido mínima si se compara con el análisis del discurso, la lexicografía o los procesos de alfabetización, que no se centran en el conocimiento de la estructura de la lengua sino, en su mayoría, en la comprensión como un mecanismo cognitivo no estrictamente linguí́stico. Por esta misma razón, la morfología ha tenido un nulo desarrollo como disciplina didáctica y no ha sido sometida a la discusión de modelos de procesamiento 
lingüístico desarrollada en las últimas décadas después del dominio imperante del estructuralismo y el generativismo.

En didáctica, el modelo de enseñanza de la morfología en las aulas españolas es de corte estructuralista, no ha cambiado a lo largo de los años, sigue basándose en la categorización de morfemas y en la descripción de procesos formativos partiendo de fundamentos historicistas y sin ninguna consideración hacia conceptos como el reanálisis (segmentaciones que hacen los hablantes en función del uso de la lengua y parámetros de similitud entre formas) o la reflexión metalingüística, metodológicamente predictora del conocimiento léxico. No ha habido una evolución en la enseñanza de la didáctica de la morfología a la par de las nuevas teorías sobre el procesamiento de la lengua que tanto pueden aportar a la didáctica. No se ha aprovechado el conocimiento sobre los procesos de adquisición del lenguaje infantil o las investigaciones con personas con trastornos linguísticos para entender cómo se puede ayudar a desarrollar la competencia morfológica de los escolares. La didáctica de la morfología ha tenido una atención mínima desde el punto de vista de la investigación lingüística y, como veremos a partir del análisis de algunos libros de texto, muy poco interés en la práctica de las aulas.

La primera cuestión que debemos plantearnos es si realmente el estudio de la morfología favorece el desarrollo de la lengua materna. Es decir, si existe relación entre el dominio de la lengua y el conocimiento de la gramática adquirido en la etapa escolar y si hay una proporción entre el esfuerzo escolar que realizan los estudiantes durante tantos años de estudio de la morfología y los beneficios de carácter expresivos que obtienen. Aquí surgen dos temas diferentes aunque ligados: el primero es el cuestionamiento del estudio de la estructura del español cuando se trata de la lengua materna y el segundo es el cuestionamiento de la metodología tradicional de enseñanza de la morfología. Vamos a realizar en primer lugar un análisis del panorama didáctico para mostrar cómo se plantea el estudio de la morfología, contrastando la discusión teórica con datos del currículum educativo de la ESO y de Bachillerato y con el desarrollo de explicaciones y actividades de morfología en algunos libros de texto. El desajuste, al menos aparentemente, que 
existe entre el planteamiento normativo y la práctica en las aulas, justifica la necesidad de investigación en didáctica de la morfología y la actualización metodológica a partir de las aportaciones de las nuevas corrientes linguiísticas que estudian el procesamiento de la morfología. En este punto se centra el objeto de este estudio que resume el título: habría que estudiar si los conocimientos de morfología que tradicionalmente se enseñan en las aulas de lengua castellana (método estructuralista) fomentan la conciencia morfológica de los estudiantes (concepto psicolingüístico vinculado a corrientes lingüísticas modernas) y facilitan la adquisición del vocabulario (fin último con funcionalidad comunicativa).

La intencionalidad del estudio de la morfología no está clara en los programas de Secundaria pero tampoco hay investigación en didáctica de la morfología que valide el cambio metodológico. Veremos cómo los objetivos de enseñanza de la morfología en esta etapa escolar son difusos y vamos a fundamentar la necesidad de investigación en un doble sentido: 1) el de validación de modelos didácticos teóricos estudiando el impacto que puede tener el conocimiento de la morfología en el desarrollo de la conciencia morfológica y esta en la estimulación del aprendizaje léxico y 2) el de validación de modelos de intervención para la enseñanza de la morfología que sean funcionales y útiles para ese desarrollo comunicativo deseado como objetivo de la enseñanza de la lengua materna.

\section{1.- La enseñanza de la morfología en la tradición y en la actualidad: "gramaticalismo" y "examinismo"}

El cuestionamiento de la enseñanza de la gramática no es nuevo. Ya Américo Castro (1922) afirma en La enseñanza del español en España (Medina Morales, 2002):

La gramática no sirve para enseñar a hablar y escribir correctamente la lengua propia, lo mismo que el estudio de la fisiología y de la acústica no enseña a montar en bicicleta [...]. Si fuera posible hacer que Cervantes analizara gramaticalmente el Quijote, no podríamos darle sino una calificación bastante mediocre. Y, sin embargo, no puede decirse que Cervantes escribiese incorrectamente el español (pp. 183-184). 
La ejemplificación de estas palabras es muy ilustrativa, así como la precisión del recelo hacia la lengua materna, dejando entrever que no existe tal duda si se trata de enseñar una lengua extranjera. Esto se ha visto reflejado en una investigación desigual en didáctica de la lengua materna y de español como lengua extranjera (ELE). La idea de que la lengua materna se aprende en un contexto de inmersión desde la niñez y que el desarrollo lingüístico de los niños no depende tanto de la intervención escolar en la asignatura de Lengua como del proceso de madurez natural potenciado por la adquisición de conocimientos de muchas materias, el aprendizaje de la lectoescritura y la interacción, debilita la productividad y rentabilidad de la enseñanza gramatical para la mejora de la comunicación.

Años después, Manuel Seco afirma en una entrevista firmada por Albalá (1969) que se "sigue confundiendo Lengua con Gramática" dando demasiada importancia al aprendizaje teórico en detrimento del práctico y que "los españoles terminan el bachillerato, hoy como ayer, sin saber expresarse con mediana precisión, tanto de forma oral como escrita. Cuando lo hacen, lo hacen con una lógica pobre, con una sintaxis pobre, con un léxico pobre." Las causas que alega siguen estando en la actualidad más vigentes que nunca: el neoanalfabetismo que nombraba Pedro Salinas y que hoy es más patente, el consumo de televisión (hoy, además, de otros soportes digitales), la falta de formación de profesorado, la rutina memorística para la preparación de exámenes... Introduce dos términos para calificar la enseñanza de la gramática que podemos aplicar a la didáctica de la morfología actual: manía gramaticalista y examinismo.

Seco considera un error grave que se crea que el idioma materno se aprende estudiando gramática y lamenta que este "gramaticalismo" esterilice los esfuerzos de profesores y alumnos. Habla de tres etapas en la adquisición de la lengua: una primera que llega hasta los 12 años (la etapa actual de Primaria) en la se adquiere la lengua de manera natural, una segunda etapa a partir de esa edad y hasta los 15 o 16 años (la etapa actual de Secundaria) donde los adolescentes tienen capacidad de reflexión para clasificar y sistematizar los hechos linguiísticos, y una tercera etapa posterior donde se perfecciona y matiza la expresión. Esta 
distinción sugiere que sería el período de la Enseñanza Secundaria el más apropiado para estudiar la morfología (no antes, no en Primaria) y desde el enfoque de la reflexión, es decir, siguiendo el planteamiento metalingüístico que desde hace décadas se viene clamando como algo moderno (Bosque, 2015). Seco propone en la misma entrevista una acción didáctica basada en situaciones comunicativas con la finalidad de "ampliar o matizar el vocabulario, utilizar determinadas series morfológicas o unas estructuras sintácticas dadas [...]." Como vemos, ya en los años sesenta del siglo pasado se criticaba la metodología gramaticalista de enseñanza de la lengua y se abogaba por la inclusión didáctica de la gramática en la teoría de la comunicación.

El término "examinismo" se refiere a cómo la enseñanza se enfoca para la superación de unos exámenes. Las pruebas de evaluación evidencian los contenidos y la metodología didáctica, pues son parte del proceso de aprendizaje y, de manera bidireccional, se prepara a los estudiantes para que superen unas pruebas y se diseñan esas pruebas en función de cómo se ha preparado a los escolares. Como veremos a continuación, los ejercicios de los libros de texto así como las pruebas de acceso a la Universidad solicitan el análisis y la clasificación de palabras y morfemas. Por tanto, suponemos que continúa hoy el aprendizaje basado en la retención memorística de definiciones y clasificaciones de la que habla Seco.

Vamos a analizar brevemente el currículum educativo en Lengua Castellana y Literatura y algunas muestras de explicaciones y ejercicios de morfología en libros de texto que reflejan cómo no ha habido cambios desde esa tradición gramaticalista y examinista de la que hablaba Seco (1969) y cómo las prescripciones normativas no se reflejan en los libros de texto ni en las pruebas de evaluación al acabar Bachillerato.

\section{1.- El estudio de la morfología en el currículum educativo de la Enseñanza Secundaria y Bachillerato}

El currículum de Lengua Castellana y Literatura en la Enseñanza Secundaria (BOCyL, 08/05/2015, p. 32164) indica en el 
bloque de Conocimiento de la lengua "la necesidad de reflexión sobre los mecanismos lingüísticos que regulan la comunicación" con la intención de utilizar el conocimiento de la lengua para su correcto uso. El desarrollo de la competencia lingüística debe construirse "a partir del conocimiento y la reflexión necesarios para apropiarse de las reglas ortográficas y gramaticales imprescindibles, para hablar, leer y escribir correctamente en todas las esferas de la vida." Se plantea la enseñanza de la gramática desde la reflexión y funcionalidad para desarrollar la competencia oral y escrita ${ }^{1}$.

Estructura los contenidos en cuatro ejes: la palabra (léxico), las relaciones gramaticales que se establecen entre palabras y grupos de palabras (morfología y sintaxis), las relaciones textuales (discurso) y las variedades linguiísticas (sociolingüística). Los dos primeros temas se centran en el estudio de la palabra, en su forma y su significado dentro del contexto de la oración y el discurso, y en las relaciones con otras palabras. Sin mención explícita, se le está dando relevancia al valor comunicativo del léxico por sus relaciones formales y semánticas con otras formas, es decir, se propone estudiar la palabra en relación con otras con las que puede compartir paradigma (si se trata de procesos flexivos) o similitudes formales o semánticas (si se trata de procesos derivativos o composición) para conocer su valor comunicativo dentro de un mensaje. Leemos entre líneas que la palabra como unidad es el signo prioritario de estudio (no se menciona el término morfema o sintagma, ni tampoco morfología o sintaxis) y que el conocimiento de las conexiones léxicas es clave para estudiar la palabra y su uso en el discurso. El planteamiento es totalmente acorde con los estudios de morfología de Bybee $(1985,1988)$, de corte conexionista y todavía imperantes en lingüística.

\footnotetext{
${ }^{1}$ Con las mismas palabras se expresa el enfoque de la enseñanza de la gramática en el currículum de la Comunidad de Madrid (BOCM, 20/05/2015, p. 72) y en el de la Junta de Andalucía (BOJA, 28/07/2016, p.174), por citar solo dos ejemplos de comunidades autónomas. Los contenidos del currículum que afectan al estudio de la morfología son los mismos tanto en la etapa de Secundaria como en la de Bachillerato en las distintas comunidades autónomas. Igualmente, los criterios de evaluación y estándares de aprendizaje.
} 
Sin embargo, esa concreción de la funcionalidad del estudio de la palabra (de la morfología) no aparece en el desarrollo de los contenidos, criterios de evaluación y estándares de aprendizaje (BOCyL 08/05/2015, pp. 32166-32179). Se menciona aquí el reconocimiento, uso y explicación de clases de palabras, elementos constitutivos de las palabras y relaciones semánticas entre palabras "para corregir errores de concordancia en textos propios y ajenos" (objetivo relativamente absurdo tratándose de la lengua materna de un adolescente) y en $4^{\circ}$ curso se dice que se observen y expliquen los valores expresivos de las distintas categorías gramaticales "en relación con la intención comunicativa del texto en que aparecen". Los contenidos, criterios y estándares de aprendizaje son repetitivos en los cuatro cursos y denotan una absoluta falta de concreción y exposición de valor práctico (y por ende, funcional para los docentes).

En el currículum de Bachillerato (tomamos como referencia ejemplificativa el del BOCyL, 08/05/2015, p. 32732) se habla de la distinción entre competencia comunicativa oral y escrita como objetivo de la materia, y se exponen con mayor precisión en los dos cursos los contenidos de morfología que han de estudiarse: se enumeran las distintas clases de palabras $\mathrm{y}$, en algunos casos, su análisis y caracterización morfológica, sintáctica y semántica, su relación con otras categorías o clases de palabras y los procesos de formación de palabras. En los criterios de evaluación sí se menciona tomar conciencia de la importancia del conocimiento gramatical para el uso correcto de la lengua. Y en los estándares, se vuelve a mencionar el conocer la morfología para evitar errores de concordancia y, en el caso del sustantivo, adjetivo y verbo, identificar la intención comunicativa del emisor (BOCyL 08/05/2015, pp. 32733-32746).

Dejando al margen la repetición de contenidos, criterios y estándares en los seis cursos y la ausencia de indicios de teoría comunicativa y de metodología didáctica, podría intuirse que tras estas afirmaciones generales está la idea de que el conocimiento de la morfología debe servir para identificar valores comunicativos y que la toma de conciencia de esos valores es un criterio evaluable. Veamos si en los libros de texto se aprecia la captación de este objetivo. 


\section{2.- El estudio de la morfología en los libros de texto y en las Pruebas de Acceso a la Universidad}

El análisis de seis libros de texto, uno de cada curso, es suficiente para ilustrar el modelo didáctico de enseñanza de la morfología. Es muy poco el espacio que se le dedica a la morfología. Su estudio se limita a clasificar palabras, dividirlas en partes y explicar el proceso de formación en el caso de la morfología léxica. En $1^{\circ}$ de ESO ya tenemos todos los contenidos de morfología que se van a enseñar a lo largo de la etapa hasta $2^{\circ}$ de Bachillerato. Las actividades reflejan en su desarrollo los criterios de evaluación en función de los objetivos y estándares de aprendizaje. En Arce et al. (2015, pp. 41-42) encontramos prácticas donde se solicita la distinción de nombres entre palabras de una lista, identificar nombres en un texto breve, clasificar sustantivos según su significado y a partir de una lista de verbos y de sufijos, formar derivados. Se está evaluando en los escolares sus conocimientos de morfología (distinguir nombres de verbos o de adjetivos, por ejemplo) y sus conocimientos de español en el ejercicio de formar derivados. En este último caso, no se puede realizar bien la actividad si no se conocen las palabras (el ejemplo con el verbo nadar para formar natación es claro, pues hay un cambio morfofonológico en la raíz y no es posible derivar correctamente si no se conoce de antemano el derivado; se da por hecho que, como es palabra frecuente, la conocen y van a formarla correctamente). Por eso, estas actividades solo sirven para que, quizás, y de forma implícita, sean conscientes de que esas palabras que conocen tienen partes. Son ejercicios que miden el conocimiento morfológico (se sabe o no se sabe) pero no son metalingüísticos desde el punto de vista procedimental, pues solo sirven para reconocer, si es el caso, lo que ya conocen.

En Pantoja Rivero et al. (2016, pp. 51-52), en $2^{\circ}$ de ESO, se incluyen unas tablas con prefijos y sufijos, su significado y algunos ejemplos, y después se solicita en las actividades que identifiquen el lexema, transformen sustantivos en adjetivos, conviertan en verbos otras palabras, formen palabras usando prefijos de negación y separen lexemas y morfemas de las palabras resaltadas en negrita en un texto. 
Son ejercicios para analizar y clasificar un poco más complejos que los de $1^{\circ}$, pero igualmente nada comunicativos. Analizar palabras dentro de un discurso no sirve en este caso de nada diferente a si esas palabras dadas estuvieran en una lista. Es un ejercicio textual disfrazado, pues no se estudia ni se aprende nada sobre la intencionalidad en el uso de esas palabras. En el mismo libro (p. 54) hay una serie de actividades de productividad y creatividad en las que se pide que formen palabras con determinados afijos y que creen compuestos a partir de algunas palabras. Implícitamente se podría intuir el objetivo didáctico que trabaja la conexión entre palabras que comparten afijos como un procedimiento para aprender léxico relacionado, pero el enfoque simple de la propuesta, sin ninguna reflexión, parece solo orientada a medir el conocimiento de palabras. Las actividades de síntesis (pp. 60, 68) son similares: identificar afijos y explicar el proceso de formación de las siguientes palabras, o subrayar los sustantivos de un texto breve.

En $3^{\circ}$ y $4^{\circ}$ de ESO, en González Bernal et al. (2015) y Bouza Alvárez et al. (2008), hay un apartado breve en cada lección dedicado a la ampliación del léxico con temas como la identidad, la igualdad, las pasiones... Pero el enfoque didáctico es igual que en los ejercicios de los libros del primer ciclo. En el del léxico de identidad de $3^{\circ}$ de ESO (p. 12) se estudia la derivación y se pide en las actividades que busquen palabras que empiecen por ego-, que indiquen el significado de palabras con auto- y, aprovechando el tema, que busquen datos sobre el mito de Narciso. El planteamiento es pobre tanto para estudiar un campo léxico como para estudiar la derivación. Pero, además, no hay más reflexión que el pensar en palabras con un prefijo, ni ningún desarrollo comunicativo ligado al estudio de la morfología ni a las relaciones semánticas (salvo que Narciso era ¿egocéntrico?). En $4^{\circ}$ de ESO se vuelve con los procesos de formación de palabras estudiados en los primeros cursos con ejercicios similares: lista de afijos, listas de palabras para identificarlos y para señalar el proceso. Aunque el enfoque según el título del apartado sea conocimiento del léxico, el proceso didáctico es de reconocimiento, es decir, de identificar lo que ya se conoce. 
En Bachillerato, en Lobato Morchón y Lahera Forteza (2015, 2016) se dedica muy poco espacio a la morfología (como en ESO) pero hay más teoría descriptiva de las clases de palabras y de los procesos formativos. Los ejercicios son idénticos a los que se plantean desde $1^{\circ}$ de ESO, con el mismo grado de exigencia en las identificaciones y el mismo nivel léxico respecto a frecuencia de uso. En los dos cursos los ejercicios requieren segmentar palabras, clasificar morfemas y señalar el proceso formativo de derivación, composición o parasíntesis. En $1^{\circ}$ los temas concluyen con un comentario de texto donde se introduce un apartado de gramática con las mismas preguntas que en los ejercicios no contextualizados: clasificar palabras del texto, identificar sus partes... Son ejercicios de reconocimiento donde se ignora la relación con la función comunicativa de la que habla el currículum. De forma aislada en el libro de $2^{\circ}$ (p. 51) se pregunta por la localización de marcas lingüísticas subjetivas y afijos valorativos en un texto brevísimo donde solo la palabra careto responde a lo demandado. Es un pequeño conato de integrar el estudio de la morfología en la teoría de la comunicación, pero insignificante en sí mismo y en el conjunto del libro de texto. Todos los ejercicios miden el conocimiento de la teoría morfológica e implican el conocimiento del léxico, pues si no hay reconocimiento de las palabras no es posible responder correctamente en la mayor parte de los casos. Por eso, nos podemos plantear si se aprende con este tipo de actividades o simplemente permiten que los hablantes sean conscientes de lo que ya saben sin entender para qué les sirve esa toma de conciencia.

La morfología estudiada en los libros de texto y su proceso de aprendizaje a través de las actividades responde exactamente al conocimiento requerido sobre el tema en las Pruebas de Acceso a la Universidad. En España estas pruebas son solo escritas (la lengua oral no se evalúa y esta puede ser una razón por la que se trabaja poco en Bachillerato) y dedican a la morfología una mínima parte del contenido gramatical (igual que los libros de texto). En los exámenes de Lengua Castellana y Literatura de las Universidades de Castilla y León, las Universidades Públicas de la Comunidad de Madrid y el distrito único de Andalucía, la prueba de comentario de texto tiene un valor mayor que la llamada prueba de lengua, que incluye sintaxis y morfología. No 
hay integración del conocimiento gramatical (preguntas de lengua) en la competencia comunicativa (comentario de texto) salvo porque, como sucedía en algunos (pocos) ejercicios de los libros de texto, las frases o palabras para analizar sintáctica o morfológicamente están extraídas del texto que han de comentar en la primera parte del examen.

La diferencia de puntuación entre Comunidades no es significativa: en Castilla y León el comentario vale 4 puntos vs. la prueba de sintaxis (2) y de morfología (1); en Madrid, comentario $(4,5)$ vs. sintaxis $(1,5)$ y morfología (1); y en Andalucía, el comentario (3) + elaboración de un texto breve (2) vs. sintaxis $(1,5)$ y morfología (1). Sin embargo, ese punto común de valoración de la prueba de morfología sí conlleva diferencias: en Castilla y León piden segmentar, describir constituyentes y clasificar las palabras por su estructura interna y categoría gramatical de dos palabras entre cuatro dadas; en Madrid, solicitan en una opción los mismos datos solo en una palabra, y en la otra opción, la pregunta es de caracterización léxica, no morfológica; en Andalucía, en una opción se pregunta solamente por cómo están formadas dos palabras, y en la otra por el significado de dos expresiones. La manera de tratar la morfología en los tres casos es totalmente tradicional y acorde con el estudio reflejado en las actividades de los libros de texto, sin embargo, en la prueba de Castilla y León la morfología tiene más peso por los datos que se piden en la descripción de las palabras y por su prevalencia en las dos opciones de examen. Aparentemente, los exámenes de las otras dos Comunidades resultan más simples en esta prueba (especialmente la andaluza) pero, en cualquier caso, en ninguna de ellas hay un enfoque comunicativo del conocimiento morfológico (ni sintáctico).

Podemos concluir que se forma en conocimiento morfológico desde $1^{\circ}$ de ESO, repitiendo los mismos contenidos y sin ninguna concreción en la funcionalidad de ese aprendizaje, pues no hay ejercicios en los libros de texto ni en las pruebas de acceso a la Universidad que reflejen la intencionalidad comunicativa de las palabras según su formación morfológica. Asimismo, tampoco hay ejercicios que permitan ampliar el vocabulario mediante una metodología reflexiva y significativa. Se forma a los estudiantes en el 
conocimiento de la morfología para que sepan identificar unidades en las palabras que ya conocen pero no hay prácticas que desarrollen el mejor uso de esas palabras en la comunicación. La instrucción morfológica que reciben no tiene aplicación consciente en el desarrollo discursivo. La didáctica imperante se centra en la descripción de la estructura morfológica, como las corrientes linguiísticas tradicionales. Por tanto, podemos afirmar que nos encontramos ante el mismo gramaticalismo y examinismo ya criticados por Seco en 1969. Pese a la intencionalidad expresada en el currículum educativo de la asignatura, ni los libros de texto ni las Pruebas de Acceso a la Universidad reflejan cambios respecto a la didáctica más tradicional.

\section{2.- Demanda de un cambio en el modelo didáctico}

Podemos justificar la necesidad de un cambio en la didáctica de la morfología basándonos en que no hay ajuste entre la normativa curricular y las prácticas de aula que hemos visto en el apartado anterior $\mathrm{y}$, también, en el anquilosamiento del modelo didáctico, que no ha sabido incorporar las aportaciones de las teorías lingüísticas recientes sobre la adquisición y organización del léxico en nuestro cerebro. No obstante, este cambio requiere el desarrollo de una investigación sólida que valide su necesidad. Habría que medir la rentabilidad que el estudio de la morfología tiene en escolares que, desde la Enseñanza Primaria, llevan formándose en los mismos contenidos. De este modo, se podría afirmar la hipótesis de que hay que modificar los prototipos fosilizados de una didáctica de la morfología de escasa utilidad. Si esto fuera así, habría que plantear 1) la necesidad de promover la práctica reflexiva de la enseñanza de la morfología en el aula de español como lengua materna y comprobar el impacto relacional entre el desarrollo de la conciencia morfológica y el desarrollo del léxico; y 2) la necesidad de desarrollar una gramática escolar inductiva, orientada al desarrollo de competencias (de carácter metalingüístico), y comprobar con pruebas de validez científica la eficacia de nuevas propuestas metodológicas. Se requiere una investigación experimental que en el caso de la lengua materna se ha reducido en español a trabajos de disponibilidad léxica con pretensión de aprovechamiento didáctico para investigaciones 
futuras (Gómez Devís, 2019), estudios empíricos con muestras reducidas (Martín Vegas, 2018; Hess Zimmermann, 2019) y trabajos de multilingüismo que han atendido a la contribución de la instrucción morfológica en procesos de alfabetización (Ramírez et al., 2010; Leonet Sieso et al., 2020). Se sigue hablando de la necesidad de un nuevo planteamiento didáctico de la morfología basado en la reflexión (Serrano Dolader, 2020) pero faltan estudios que prueben que una nueva metodología metalingüística potenciará la funcionalidad de la didáctica con impactos concretos en el desarrollo competencial.

La investigación experimental necesaria para probar la eficacia didáctica y, en función de los resultados, pautar modelos, ha tenido más interés en ELE. Los profesores universitarios de ELE son generalmente investigadores y ellos mismos pueden realizar las pruebas con sus estudiantes adultos. Por el contrario, es difícil realizar experimentaciones masivas en la Enseñanza Secundaria, pues se requiere intervención externa y permisos más complejos al tratarse de menores. Este aspecto práctico, ligado a otros intereses, muestra un vacío en la investigación que esperemos que pronto pueda impulsarse.

\section{3.- Claves para un modelo didáctico de la morfología}

El estudio de Serrano Dolader (2018) sobre la enseñanza de la morfología en ELE tiene el planteamiento didáctico que se viene demandando desde hace décadas para la lengua materna. Diseña actividades de reflexión morfológica para entender el léxico conocido, comprender el nuevo e inferir reglas. Combina las explicaciones descriptivas con actividades que promueven la conciencia morfológica, pues no solo comprueban conocimientos adquiridos, sino que permiten hacer deducciones, crear nuevas formas y pensar sobre palabras posibles o imposibles en la lengua. Su metodología se centra en el proceso de adquisición del léxico considerando implícitamente el principio de organización asociativa del léxico como vía eficaz para la adquisición. Esta obra es un corpus modélico de actividades para adaptar a las aulas de Secundaria. Liga el conocimiento de la morfología con el desarrollo de conciencia metalingüística en función 
del aprendizaje de vocabulario, pues el objetivo es que los estudiantes comprendan mejor las palabras y extiendan las redes de su léxico en español. Para la elaboración de las propuestas sigue las fases del proceso de adquisición del vocabulario destacadas en investigaciones precedentes: actividades orientadas al reconocimiento de la forma identificando los constituyentes en otras formas relacionadas, actividades orientadas a deducir el significado a partir de otras palabras relacionadas morfosemánticamente más o menos transparentes, y actividades para trabajar las restricciones sistemáticas morfológicas, semánticas, sintácticas y otras de carácter normativo. Son parte de las estrategias de aprendizaje de vocabulario de Graves (2006) verificadas en estudios de instrucción léxica con angloparlantes. Además, se adhiere al principio de funcionalidad comunicativa que veíamos que indica el currículum educativo, pues se insiste en la recomendación de trabajar siempre con palabras contextualizas, no aisladas.

La pregunta es, ¿por qué no se trabajan este tipo de actividades de morfología en Secundaria y Bachillerato? Insistimos en la idea central de este estudio: falta investigación lingüística y didáctica que avale la necesidad del cambio y es necesaria una formación del profesorado de Secundaria que mentalice sobre la enseñanza funcional de la morfología y ponga en marcha un cambio de estrategia.

Vamos a ver a continuación la aportación que algunos estudios experimentales de medición de conciencia morfológica en ELE y niños con trastornos del lenguaje pueden aportar a la fundamentación de un nuevo modelo de enseñanza de la morfología en Secundaria. Asimismo, ideas relevantes de corrientes linguísticas modernas sobre la organización de la morfología en el léxico mental nos ayudarán a definir las claves de un modelo didáctico de la morfología que integre los tres objetivos "difusos" que dan título a este trabajo.

\section{1.- Aportaciones de estudios psicolinguiísticos sobre conciencia morfológica}

El concepto de conciencia morfológica no ha sido siempre bien entendido o definido en estudios realizados por psicólogos españoles 
que centran su interés en procesos de lectoescritura, que es el ámbito donde más se ha estudiado. Tres ideas principales sobre el desarrollo de la morphological awareness, muy trabajada en investigaciones de todo tipo sobre el inglés, nos pueden aportar luz a la didáctica de la morfología en Secundaria: a) su desarrollo secuencial, b) su mayor desarrollo en relación con el volumen léxico y c) la potencialidad de la intervención escolar para mejorar sus efectos.

En el lenguaje infantil hay un desarrollo secuencial de la conciencia morfológica derivativa (Tyler y Nagy, 1989) que es muy interesante tanto para diseñar trabajos experimentales como para elaborar modelos didácticos. En una primera etapa hay un conocimiento relacional entre palabras que conduce a creaciones posibles en la lengua aunque en algún caso inexistentes (por ejemplo, * globero 'el que vende globos'; Martín Vegas, 2015); en una segunda fase, el conocimiento se amplía a la sintaxis y se es consciente de que hay sufijos que forman sustantivos y otros adjetivos, por ejemplo (-miento, -able); y en una tercera fase, el conocimiento distribucional comprende el origen de los derivados (-miento y -able crean derivados a partir de verbos).

Esta secuenciación ha sido probada en Sánchez Gutiérrez (2020) con un grupo de estudiantes de ELE que se sometió a experimentación con pruebas de recepción y producción, antes y después de un período breve de intervención. El impacto de la intervención fue mayor en el grupo crítico que en el de control, lo que valida el entrenamiento didáctico. Sin embargo, lo más relevante desde nuestro punto de vista es que los resultados mostraron que el entrenamiento morfológico no tiene un impacto significante en el desarrollo de la morfología relacional en la parte productiva pero sí hay efecto, aunque no muy grande, en la parte distribucional de esta tarea. Esto avala la secuenciación de la conciencia morfológica que mencionan Tyler y Nagy (1989) y habría que tenerlo en cuenta para nivelar la tipología de ejercicios en los cursos de Secundaria. Igualmente, se probó que la mejora en la morfología relacional fue superior en los cursos más avanzados, lo que indica que los estudiantes con más vocabulario son capaces de establecer mayor tipo de relaciones morfológicas. 
Enlazamos de este modo con la segunda idea de que la conciencia morfológica es superior cuando el caudal léxico es mayor. Esta idea está avalada también por estudios como el de Mendoza (2001), que muestra que el desarrollo léxico es el principal predictor del reconocimiento morfológico en niños con Trastorno Específico del Lenguaje (TEL). Estos niños presentan deficiencias ligeras en distintas áreas del lenguaje pero severas en la comprensión y producción de la morfología; les cuesta el aprendizaje paradigmático (flexión verbal) e inducir la regla del plural en nombres, por ejemplo (aprenden pelota y pelotas como dos palabras distintas). Se confirma la hipótesis de la masa crítica de Marchman y Bates (1994) y el U-shaped learning de los verbos en inglés de Rumelhart y McClelland (1986): los niños con TEL tienden a sobrerregularizar a partir de los 24 meses los verbos irregulares que antes formaban correctamente porque ya usan al menos 60 verbos. La conciencia morfológica que permite inferir reglas se desarrolla más cuando el volumen léxico es mayor. De este modo, es en la etapa de la adolescencia cuando con un ritmo de adquisición del vocabulario más rico (Hess Zimmermann, 2019, p. 194) se puede aprovechar mejor el conocimiento morfológico para el desarrollo metalingüístico en beneficio del aprendizaje léxico.

Los estudios citados valoran la intervención escolar en el desarrollo de la conciencia morfológica, pero otra cuestión que nos planteamos es cuál es el momento más adecuado para trabajar la morfología en las aulas de español. El currículum escolar es muy repetitivo en contenidos de Lengua en todas las etapas (Primaria, Secundaria y Bachillerato) y parece una muestra de ineficiencia didáctica el hecho de que se estudie morfología desde $3^{\circ}$ de Primaria y los mismos contenidos básicos se repitan en los programas de todos los cursos de Secundaria y Bachillerato. Acosta Rodríguez et al. (2020) demuestran mediante un programa de intervención para que presten atención a la morfología niños con Trastornos en el Desarrollo del Lenguaje (TDL) y con Desarrollo Típico (DT) de una media de 6 años de edad, que la intervención funciona más aún en niveles con dificultades. Los alumnos con TD presentan una mejora mayor después de la intervención que los de DT: su capacidad para entender significados de palabras complejas con estructuras morfológicas 
similares a las estudiadas supone un avance mayor que la de niños de DT. Podría deducirse de estos resultados que la intervención temprana es conveniente pero, ciertamente, este experimento se centra más bien en el reconocimiento de la morfología flexiva. Por eso, en el caso de la morfología léxica es probable que la intervención sea más eficaz cuando los alumnos tengan ya un vocabulario rico que les permita, de manera instrínseca, tener un mayor desarrollo de la conciencia morfológica y poder aprovechar mejor todo tipo de conocimiento de la morfología con fines metalingüísticos. No hay investigaciones sobre este tema y es una tarea pendiente prioritaria para la reorganización de contenidos del currículum educativo.

Podemos concluir que sí hay evidencia de que la enseñanza explícita sobre la naturaleza morfológica de las palabras mejora su aprendizaje, así como la transferencia de conocimiento a palabras similares, pero queda por investigar cuándo es la mejor etapa escolar para iniciar la instrucción.

\section{2.- Aportaciones de las teorías unitarias del procesamiento de la morfología}

Los estudios tradicionales de adquisición de la morfología (principalmente centrados en la morfología flexiva y, de producción prolífica, en la formación del pasado del verbo en inglés) son dualistas: suponen una transición entre los procesos de aprendizaje del léxico de memoria, a la inferencia de la regla y su aplicación por defecto hasta la identificación de excepciones. De esta manera, los hablantes llegan a ser conscientes de que hay palabras cuya formación se somete a unas reglas y otras que hay que aprender de memoria. En didáctica, esto se traduce en la enseñanza de la segmentación en lexemas y morfemas, reglas de formación de palabras, flexión regular, irregular y supleción (como hemos visto en el apartado 1.2.).

Los modelos unitarios de representación de la morfología (Bybee, 1985, 1988; conexionismo) explican esta evidencia experimental en el aprendizaje de la morfología regular e irregular a través de un solo mecanismo con cambios cuantitativos graduales; es 
decir, no es que haya dos mecanismos distintos (la memoria y el procesamiento por regla), sino que las distintas fases de aprendizaje se deben a la reorganización de la propia red asociativa de palabras como consecuencia de la introducción de más formas en el sistema (Martín Vegas, 2006, pp. 174-178). La repercusión didáctica de estos modelos afecta a conceptos como el de reanálisis morfológico (frente a la segmentación historicista; Martín Vegas, 2005) y redes asociativas léxicas (conexiones formales y semánticas muy relevantes en el estudio de familias de palabras), así como a nuevos planteamientos metodológicos que tengan en cuenta el rendimiento y la productividad de las formas compartidas (las similitudes formales y semánticas entre palabras que comparten afijos o lexemas).

Las conexiones léxicas, que cuando se reconocen permiten el desarrollo de la conciencia morfológica relacional, se activan en función de algunos predictores reguladores del que debe ser modelo didáctico: a) la transparencia formal y semántica de cada lexema o afijo, b) su biunivocidad (los afijos donde hay una correspondencia de "una forma = un significado" son predecibles y más fáciles de interpretar), c) la frecuencia de los modelos morfológicos (el rendimiento de los afijos o de los lexemas formando familias) y d) la frecuencia de uso de las palabras (las más usuales se aprenden antes). Estos cuatro pilares deben regular, en nuestra opinión, el diseño de ejercicios de morfología con el fin de activar gradualmente la conciencia relacional, sintáctica y distributiva de la morfología, en terminología de Tyler y Nagy (1989).

Teniendo siempre en cuenta que cualquier modelo didáctico innovador debe aplicar una metodología que genere mejores resultados con menos esfuerzo, resumimos las pautas que ha de seguir un modelo didáctico para un aprendizaje rentable de la morfología:

a) impulsar la reflexión metalingüística sin complejidad teórica y terminológica;

b) relacionar los signos lingüísticos según sus conexiones semánticas y formales emulando la organización del léxico en nuestro cerebro según esquemas modulares interconectados por rasgos de similitud; 
c) implementar una metodología de aprendizaje significativo para promover la reflexión en el alumno, pues solo a través de una actitud indagadora se podrá conseguir esta toma de conciencia pretendida con el estudio de la morfología;

d) nivelar las actividades didácticas en función de la madurez lingüística de los alumnos y de la fuerza léxica, en terminología de Bybee. Cada término tiene una relevancia en la lengua según su frecuencia de uso (token frequency) y la frecuencia del modelo al que pertenezca (type frequency). Los dos tipos de frecuencia confluyen para nivelar la didáctica del léxico: primero debe enseñarse el léxico más usual o cercano al mundo circundante de los aprendices de la lengua (independientemente del nivel de conexiones morfológicas) y, después, el léxico perteneciente a modelos menos frecuentes o el referido a campos más especializados ${ }^{2}$.

\section{3.- Propuesta modélica}

Considerando las aportaciones de las teorías lingüísticas y los planteamientos didácticos de la gramática en particular y de la pedagogía en general, en Martín Vegas (2019) se diseñan actividades prototípicas de morfología para desarrollar en Secundaria. Se han considerado el contexto, la frecuencia y las conexiones semánticas y formales como principales factores que favorecen la conciencia de la palabra con el objetivo de dirigir todo el conocimiento morfológico adquirido como motor metalingüístico para el aprendizaje del léxico. Esa necesidad de impulsar la reflexión para la enseñanza de la morfología se plantea mediante una metodología de investigaciónacción en el aula: se propone una cuestión, se ofrecen recursos para la investigación (lematizador, diccionario inverso) y se solicita la interpretación o construcción de mensajes que activen lo aprendido. La

\footnotetext{
${ }^{2}$ Gil Laforga (2019) plantea "acotar, reducir y graduar" los contenidos de morfología que se estudian en Secundaria atendiendo, dentro de una perspectiva sincrónica, a criterios de transparencia morfosemántica que afectan a la identificación, recurrencia e intercambiabilidad de morfemas. Estos criterios están implícitos en la fuerza léxica (y los dos tipos de frecuencia) que estamos señalando como claves para un modelo didáctico de la morfología.
} 
consideración de la gramática implícita del alumno y la contextualización del estudio de la morfología son pilares metodológicos básicos (por ejemplo, se deben estudiar relaciones afijales o los procesos de nominalización en corpus extraídos a partir de www.todalaprensa.com, http://corpus.rae.es/creanet.html).

La integración de la reflexión morfológica en la didáctica debe enfocarse hacia el aprendizaje léxico con actividades de comprensión y producción discursiva. Presentamos un ejemplo en la tabla 1.

\section{Tabla 1}

Modelo de actividad. La nominalización

Contenido morfológico.

Teoría.
Estructura de la palabra.

Identificar sufijos.

Reconocer cambio de categoría.

caer - caída

variar-variación

adoptar-adopción

comenzar-comienzo
Uso de las nominalizaciones.

Comprensión en contexto.

Conciencia morfológica.

Aprendizaje léxico.

Práctica.
Titulares de prensa: "La adopción en China ha descendido en los últimos años"

Índice: "La variación lingüística"

Determinación de un tema: "La caída de los precios de los pisos"

Enunciado explicativo: "La crisis ha provocado la caída de los precios de los pisos"

Cronología: "12 de febrero: comienzo del curso de innovación en lengua"

Procedimiento de cohesión: "Una manera de tener hijos es adoptándolos. La adopción..."

Actividades de reflexión morfológica.

Integración de conocimiento y conciencia morfológicos para el desarrollo léxico.
Integrar dos enunciados dados en uno solo (Se va a reducir el sueldo a los trabajadores. Los trabajadores están inquietos. $\rightarrow$ La reducción del sueldo es la primera inquietud de los trabajadores. Los trabajadores temen...)

Completar huecos en un texto con nominalizaciones como procedimiento de cohesión.

Comprensión lectora: identificar tema, agregar subtítulos, hacer esquema...

Composición de textos: componer titulares, hacer cronologías...

Fuente: elaboración propia 
En la tabla 1 vemos los tres objetivos de estudio de la morfología ejemplificados en su desarrollo didáctico. El conocimiento de la morfología, que a su vez favorece la conciencia morfológica, sirve para desarrollar mejor la expresividad léxica necesaria para actos linguísticos concretos. La concatenación de los tres objetivos se orienta hacia la intención común de servir al mejor uso de la lengua, como indica el currículum educativo sobre la funcionalidad de la enseñanza de la gramática. En el ejemplo concreto del estudio del proceso de la nominalización de la tabla 1, el conocimiento de la estructura de nombres derivados deverbales (contenido morfológico, teoría) se pone en práctica con el reconocimiento de esos derivados en contextos lingüísticos reales (titulares de prensa, índices...) comprendiendo su valor comunicativo. Esta toma de conciencia de la morfología de la palabra permite comprender su funcionalidad estilística (contextos de uso, valor discursivo...) y permite aprender a emplear ese mecanismo morfológico a partir de actividades de reflexión como, por ejemplo, integrar dos enunciados en uno o componer titulares. De este modo, se integra el conocimiento de la morfología en el uso correcto del léxico dentro del discurso respondiendo al fin último del aprendizaje de la gramática, el desarrollo de la comunicación lingüística.

\section{Conclusiones}

El conocimiento de la morfología, la conciencia morfológica y el aprendizaje léxico son tres objetivos difusos en la Enseñanza Secundaria porque ni el currículum precisa en su recurrencia extrema qué implica la reflexión de la lengua, cómo ha de generarse ni cómo ha de integrarse la morfología en la teoría comunicativa, ni los libros de texto reflejan ni concretan ese propósito no precisado en el currículum. La didáctica de la morfología no ha superado el gramaticalismo y el examinismo que empañan la didáctica de la gramática desde siempre y los planteamientos de cambio tan divulgados en los estudios actuales sobre el tema siguen sin llevarse a la práctica $\mathrm{y}$, además, sin ser sometidos a una investigación previa que verifique su necesidad con especificación. 
Habría que estudiar la rentabilidad de la didáctica de la morfología en Secundaria para saber si es necesario hacer cambios en la enseñanza y en qué dirección. Interesa, en primer lugar, medir mediante experimentación psicolingüística el impacto que la conciencia morfológica (léxica) tiene en el conocimiento del vocabulario de los adolescentes, -que llevan años recibiendo instrucción en morfología y ya tienen un volumen léxico considerable-, para valorar la necesidad de cambios. Si el impacto fuera bajo, deduciríamos que toda la instrucción recibida a lo largo de su etapa de escolarización no ha sido favorable. En segundo lugar, y en función de los resultados, habría que desarrollar modelos de intervención y validar empíricamente qué actividades enfocadas a la toma de conciencia de los procedimientos de formación de palabras permiten decodificar mejor otras palabras relacionadas, integrar ese aprendizaje léxico en ejercicios de creatividad discursiva y desarrollar procesos de aprendizaje autónomos en el marco de la neurodidáctica. Es necesario "poner ciencia sobre la mesa" e investigar con procedimientos de validez científica (no especulativa) qué carga de entrenamiento (instrucción) se necesita implementar en las aulas para llegar a resultados que se puedan medir y, consecuentemente, afirmar que el estudio de la morfología a partir de un determinado nivel, con una metodología conveniente y ocupando un espacio determinado en el programa de la asignatura, favorece que los estudiantes progresen significativamente en su desarrollo comunicativo.

Los datos de una investigación empírica en morfología permitirán modular programaciones de aula y desarrollar procesos evaluables que se ajusten en función del nivel de los estudiantes. La formación del profesorado de Secundaria depende de investigaciones externas absolutamente necesarias para saber, por fin, cómo transformar las capacidades de los estudiantes en competencias mediante dinámicas concretas. Hay que probar si la mayor riqueza léxica de los adolescentes es causa o consecuencia de su conocimiento morfológico. El objetivo de la didáctica de la morfología debe ser el afianzamiento de la conciencia lingüística con el fin de estimular el desarrollo léxico. Si no es así, no saldremos del gramaticalismo, en la acepción del término peor y más denostada por los estudiantes. 
En este estudio hemos presentado las claves para un modelo didáctico de la morfología aplicando conclusiones de estudios psicolingüísticos sobre conciencia morfológica (desarrollo secuencial, relación con el volumen léxico y potencialidad de programas de intervención) y los fundamentos de las teorías unitarias sobre el procesamiento de la morfología. La didáctica de la morfología debe modernizarse, integrarse en los avances de las teorías linguísticas y fundamentarse en una investigación empírica sólida que pueda experimentar en la práctica modelos de intervención rentables. De esta manera, los tres objetivos "difusos" en la Enseñanza Secundaria serán manifiestos y globales.

\section{Referencias bibliográficas}

Acosta Rodríguez, V. M., Hernández Expósito, S., y Ramírez Santana, G. A. (2020). Intervención temprana en conciencia morfológica en alumnado con Trastornos en el Desarrollo del Lenguaje. Revista de Educación, 390, 57-73. doi: https://doi.org/10.4438/1988592X-RE-2020-390-465

Albalá, A. (1969). La enseñanza del español en España. Entrevista a Manuel Seco. Informaciones, 26/11/1969.

Arce, M., López, L., Miret, P., y Mola, L. (2015). Lengua castellana y literatura. 1 ESO Vigía. Barcelona: Teide.

BOCM (20/05/2015). DECRETO 48/2015, de 14 de mayo, del Consejo de Gobierno, por el que se establece para la Comunidad de Madrid el currículo de la Educación Secundaria Obligatoria.

BOCyL (08/05/2015). ORDEN EDU/362/2015, de 4 de mayo, por la que se establece el currículo y se regula la implantación, evaluación y desarrollo de la educación secundaria obligatoria en la Comunidad de Castilla y León.

BOCyL (08/05/2015). ORDEN EDU/363/2015, de 4 de mayo, por la que se establece el currículo y se regula la implantación, evaluación y desarrollo del bachillerato en la Comunidad de Castilla y León.

BOJA (28/07/2016). Orden de 14 de julio de 2016, por la que se desarrolla el currículo correspondiente a la Educación Secundaria 
Obligatoria en la Comunidad Autónoma de Andalucía, se regulan determinados aspectos de la atención a la diversidad y se establece la ordenación de la evaluación del proceso de aprendizaje del alumnado.

Bosque, I. (2015). Nuevas reflexiones sobre la enseñanza de la gramática. Actitudes frente a contenidos. II Jornadas GrOC. Barcelona: UAB, 5 de febrero de 2015. Recuperado de http://www.edugroc.com/castellano/jornadas/jornadas-2015/.

Bouza Álvarez, M. T., González Bernal, J. M., Pérez Fuente, J. L., y Romeu Rodríguez, A. (2008). Lengua Castellana y Literatura. $4^{\text {o }}$ Secundaria. Serie Cota. Madrid: Oxford Educación.

Bybee, J. L. (1985). Morphology. A study of the relation between meaning and form. Amsterdam: John Benjamins.

Bybee, J. L. (1988). Morphology as lexical organization. En M. Hammond y M. Noonan (Eds.), Theoretical morphology. Approaches in modern linguistics (pp.119-141). San Diego: Academic Press.

Bybee, J. L. (1991). Natural morphology: the organization of paradigms and language acquisition. En T. Huebner y C. A. Ferguson (Eds.), Crosscurrents in second language acquisition and linguistic theories (pp.67-92). Amsterdam/Philadelphia: John Benjamins.

Bybee, J. L. (1995). Diachronic and typological properties of morphology and their implications for representation. En L. B. Feldman (Ed.), Morphological aspects of language processing (pp.225-246). Hillsdale, NJ/Hove: Lawrence Erlbaum.

Dalton-Puffer, C. (1993). A diachronic view on morphological productivity: the case of English deverbal adjectives. En L. Tonelli y W. U. Dressler (Eds.), Natural morphology. Perspectives for the nineties. Selected papers from the workshop at the fifth international morphology meeting (Krems, 4-9 july 1992) (pp.157-168). Padua: Unipress.

Daugherty, K. G., y Hare, M. (1994). What's in a rule? The past tense by some other name might be called a connectionist net. En M. C. Mozer et al. (Eds.), Proceedings of the 1993 Connectionist Models Summer School (pp.149-156). Hillsdale, NJ: Lawrence Erlbaum Associates. 
Gasser, M. (1994). Modularity in a connectionist model of morphology acquisition. International Conference on Computational Linguistics, 15, 214-220.

Gasser, M. (1997). Transfer in a connectionist model of the acquisition of morphology. En G. Booij y J. von Marle (Eds.), Yearbook of Morphology 1996 (pp.97-115). Dordrecht: Kluwer Academic Publishers.

Gil Laforga, I. (2019). El análisis morfológico en el aula. Perspectiva, contenidos y actividades. ReGrOC: Revista de Gramática Orientada a las Competencias, 2(1), 43-66. doi: https://doi.org/10.5565/rev/regroc.29

Gómez Devís, M. B. (2019). A propósito de las redes semánticas en el léxico disponible de escolares de primero de Educación Primaria. Ogigia. Revista electrónica de estudios hispánicos, 25, 165-183. doi: https://doi.org/10.24197/ogigia.25.2019.165-183

González Bernal, J. M., González Lavado, B., y Portugal Pardo, J. (2015). Lengua castellana y literatura. 3 ESO. Madrid: Oxford Educación.

Graves, M. F. (2006). The vocabulary book. Nueva York: Teachers College Press.

Hess Zimmermann, K. (2019). Pensar sobre la Morfología de las Palabras: un Proyecto Didáctico para el Desarrollo de Vocabulario en la Escuela Secundaria. Revista Iberoamericana De Evaluación Educativa, 12(2), 193-215. doi: https://doi.org/10.15366/riee2019.12.2.010

Leonet Sieso, O., Cenoz Iragui, M. J., y Gorter, D. (2020). Developing morphological awareness across languages: translanguaging pedagogies in third language adquisition. Languages awareness, 29(1), 41-59. doi: https://doi.org/10.1080/09658416.2019.1688338

Lobato Morchón, R., y Lahera Forteza, A. (2015). Lengua Castellana y Literatura. Bachillerato 1. Madrid: Oxford Educación.

Lobato Morchón, R., y Lahera Forteza, A. (2016). Lengua Castellana y Literatura. Bachillerato 2. Madrid: Oxford Educación.

Marchman, V. A., y Bates, E. (1994). Continuity in lexical and morphological development: A test of the critical mass 
hypothesis. Journal of Child Language, 2(2), 339-366. doi: https://doi.org/10.1017/S0305000900009302

Martín Vegas, R. A. (2005). ¿Segmentación o reanálisis? Formaciones con el sufijo -idad o *-abilidad, *-icidad, *-eidad. Moenia. Revista Lucense de Lingüística \& Literatura, 2, 269-281. http://hdl.handle.net/10347/5736

Martín Vegas, R. A. (2006). Introducción a la morfofonología contemporánea. Munich: LINCOM Handbooks in Linguistics 21.

Martín Vegas, R. A. (2007). Morfofonología histórica del español. Estudio de las alternancias /jél - /el, /wél - /o/ yc - /g/. Munich: LINCOM Studies in Romance Linguistics 51.

Martín Vegas, R. A. (2015). Desarrollo del lenguaje en la educación infantil. Madrid: Editorial Síntesis.

Martín Vegas, R. A. (2018). Modelos de aprendizaje léxico basados en la morfología derivativa. Rilce. Revista de Filología Hispánica, 34(1), 262-285. doi: https://doi.org/10.15581/008.34.1.26285

Martín Vegas, R. A. (2019). La conciencia lingüística de la palabra. En M. Carmen Fernández López y M. Martí Sánchez (Eds.), El desarrollo de la conciencia lingüística en aprendices de español (pp.37-83). Alcalá de Henares: Servicio de Publicaciones de la Universidad de Alcalá.

Medina Morales, L. P. (2002). ¿Para qué aprender gramática en la escuela? Puentes entre la abstracción del análisis y la comunicación cotidiana. Onomázein, 7, 183-212.

Mendoza, E. (2001). Los problemas gramaticales en el TEL. En E. Mendoza Lara (Coord.), Trastorno específico del lenguaje (TEL) (pp.85-113). Madrid: Pirámide.

Pantoja Rivero, J. C., Espí Jimeno, L., Gónzález Gallego, B., Mateos Danaire, E., Río Luelmo, M. del, y Sales Desi, E. (2016). Lengua Castellana y Literatura. $2^{\circ}$ ESO. Madrid: McGraw Hill Education.

Pinker, S., y Prince, A. (1988). On language and connectionism: Analysis of a parallel distributed processing model of language acquisition. Cognition, 28, 73-193. doi: https://doi.org/10.1016/0010-0277(88)90032-7 
Plunkett, K., y Marchman, V. (1991). U-shaped learning and frequency effects in a multi-layered perceptron: Implications for child language acquisition. Cognition, 38, 3-102. doi: https://doi.org/10.1016/0010-0277(91)90022-V

Plunkett, K., y Marchman, V. (1993). From rote learning to system building: acquiring verb morphology in children and connectionist nets. Cognition, 48, 21-69. doi : https://doi.org/10.1016/0010-0277(93)90057-3

Ramírez, G., Chen-Bumgardner, B., Geva, E., y Kiefer, H. (2010). Morphological awareness in Spanish-English bilingual children: Within and cross-language effects on word reading. Reading and Writing: An Interdisciplinary Journal, 23, 337-358. doi: https://doi.org/10.1007/s11145-009-9203-9

Rumelhart, D. E., y McClelland, J. L. (1986). On learning the past tenses of English verbs. En J. L. McClelland y D. E. Rumelhart (Eds.), Parallel distributed processing: Explorations in the microstructure of cognition. Vol. 2: Psychological and biological models (pp.216-271). Cambridge, Mass.: The MIT Press.

Sánchez Gutiérrez, C. H. (2020). Morphological awareness in L2 Spanish: what is learned incidentally and what requires explicit teaching? Revista Internacional de Lenguas Extranjeras, 13. doi: http://doi.org/10.17345/rile13.2878

Sánchez Miret, F, Koliadis, A., y Dressler, W. U. (1998). Connectionism vs. rules in diachronic morphology. Folia linguistica histórica, 18, 149-182. doi: https://doi.org/10.1515/flih.1997.18.1-2.149

Serrano Dolader, D. (2018). Formación de palabras y enseñanza del español LE/L2. London/New York: Routledge.

Serrano Dolader, D. (2020). La formación de palabras en el aula: observar, pensar y reflexionar. Revista de Gramática Orientada a las Competencias, 3(1). doi: https://doi.org/10.5565/rev/regroc.61

Tyler, A., y Nagy, W. (1989). The acquisition of English derivational morphology. Journal of Memory and Language, 28(6), 649-667. doi: https://doi.org/10.1016/0749-596X(89)90002-8 
Tejuelo, nº 35.1 (2022), págs. 107-136. Conocimiento de la morfología...

136 | Págin a 